\title{
Unilateral hemispheric primary angiitis of the central nervous system
}

\author{
Michael G. Ho • Wanxing Chai · Harry V. Vinters • \\ Gasser Hathout - Shri Mishra - Catherine Yim • \\ Miguel Valdes-Sueiras $\cdot$ Robert Nishimura
}

Received: 6 January 2011/Revised: 1 March 2011/ Accepted: 3 March 2011/Published online: 18 March 2011

(C) The Author(s) 2011. This article is published with open access at Springerlink.com

Dear Sirs,

A 52-year-old Hispanic man was admitted to hospital with a history of seizures, right sided hemiparesis, and new onset of aphasia. He complained of seizures that began 3 years prior which were characterized by 5 min episodes of right leg twitching, spreading to his right arm. The patient had no known risk factors for seizures, and the addition of topiramate had reduced his seizure frequency to about once every 2-3 days. A previous MRI of his brain showed nonspecific fluid attenuation inversion recovery (FLAIR) hyperintensities in the left cerebral hemisphere; however, his EEG was normal. In the past, the patient had a brain biopsy for a workup of the FLAIR hyperintensities in the left hemisphere, which was inconclusive due to

M. G. Ho · W. Chai · H. V. Vinters - M. Valdes-Sueiras ·

R. Nishimura $(\bowtie)$

Department of Neurology, David Geffen School of Medicine, University of California, Los Angeles, Los Angeles, CA, USA e-mail: rnishimu@ucla.edu

\section{H. V. Vinters}

Department of Pathology and Laboratory Medicine (Neuropathology), David Geffen School of Medicine,

University of California, Los Angeles, Los Angeles, USA

G. Hathout · C. Yim

Department of Radiology (Neuroradiology), Olive View-UCLA

Medical Center, Los Angeles, CA, USA

S. Mishra $\cdot$ M. Valdes-Sueiras · R. Nishimura

Department of Neurology, Olive View-UCLA Medical Center, Los Angeles, CA, USA inadequate tissue sampling. His neurological examination was remarkable for a global aphasia, 4/5 strength on the right upper and lower extremities, and a right sided spastic gait with right sided internal circumduction of the foot.

MRI scan of the brain showed extensive fluid attenuation inversion recovery (FLAIR) hyperintensities restricted to the left cerebral hemisphere, with multiple foci of nodular and ring enhancement, along with involvement of the left calvarium (Fig. 1a-c). Serum rheumatologic and infectious studies were negative outside of a positive serum Bartonella antibody titer. Cerebrospinal fluid results were unremarkable. Since the patient had a history of working with farm animals, he was treated empirically with doxycycline for 4 weeks, but with no noticeable improvement. MRI scans of the brain over the next few months showed progression of the FLAIR hyperintensities in the left hemisphere. Since the patient was a recent immigrant from Mexico, he was also treated with RIPE (rifampin, isoniazid, pyrazinamide and ethambutol) therapy for presumed tuberculosis with no noticeable improvement.

After a month, the patient presented to another hospital because of worsening aphasia and headaches. An MRI scan showed further progression of the left hemispheric lesions. Four-vessel cerebral angiogram was notable for a small left middle cerebral artery saccular aneurysm and a right pericallosal anterior cerebral artery aneurysm, which were both surgically clipped, but no beading or stensoses were visualized. He then had a left frontal stereotactic brain biopsy performed revealing dense perivascular chronic inflammation with mixed $\mathrm{T}$ and $\mathrm{B}$ lymphocytes, along with reactive astrocytosis in surrounding brain. The inflammatory infiltrates were sharply demarcated from the surrounding brain and did not extend into the surrounding neuroglial tissue. The findings were consistent with 

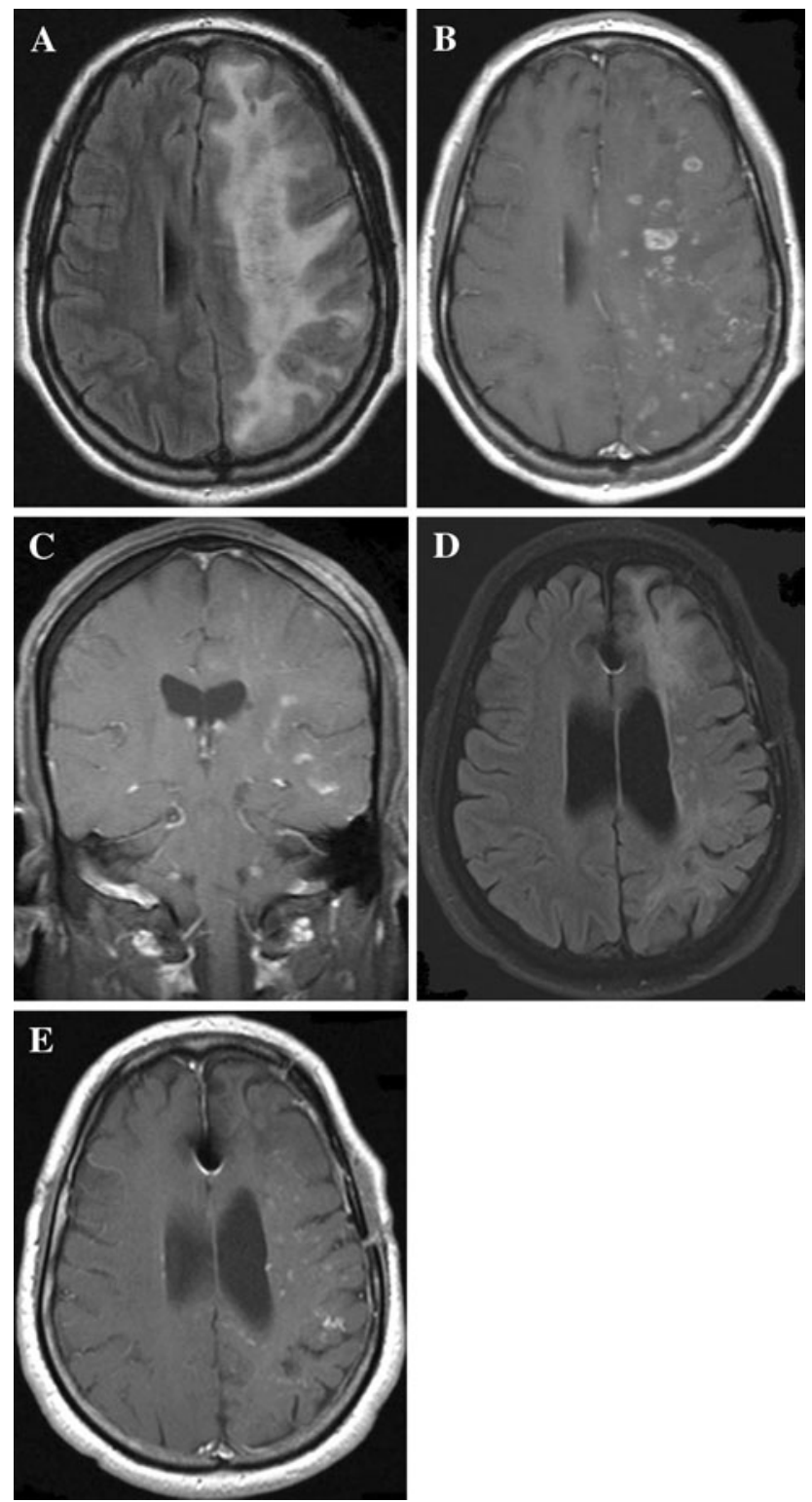

Fig. 1 MRI brain revealing extensive fluid attenuated inversion recovery (FLAIR) lesions restricted to the left cerebral hemisphere (a), with multiple foci of nodular and ring enhancement (b), along with involvement of the left calvarium (c). After treatment with glucocorticoids and cyclophosphamide, the repeated MRI showed resolution of most of the FLAIR hyperintensities in the left cerebral hemisphere (d), and resolution of most of the enhancing areas on T1 post contrast imaging (e), along with interval aneurysm clipping of the left anterior cerebral artery aneurysm and left middle cerebral artery aneurysm

primary angiitis of the CNS (PACNS) (Fig. 2a-f). The patient returned to our care and was treated with corticosteroids and cyclophosphamide. Within days after initial treatment the patient noticed improvement of speech and strength.
At a follow up visit 3 months later, the patient reported near complete resolution of his aphasia, seizures and right sided hemiparesis. He has not had a seizure in over 6 months, and a follow up MRI brain revealed significant improvement of the FLAIR and significantly decreased areas of enhancement in the left hemisphere (Fig. 1d, e).

Our case is the first in the literature that reports a slowly progressive, single hemispheric involvement of PACNS. The patient's focal, unilateral lesion on MRI and slow indolent course initially raised the question of infection, tumor, or even a progressive demyelinating disease. In addition, the unusual manifestation of focal involvement of the overlying calvarium raised the possibility of granulomatous disease, such as vasculitis occurring in the setting of tuberculous meningitis. However, the diagnostic studies for tuberculosis including tuberculin skin test, sputum cultures, and cerebrospinal fluid cultures were all negative, and the MRI did not reveal the basilar meningeal thickening that might suggest tuberculous meningitis.

Our patient required two brain biopsies before there was adequate sampling of the tissue and vessels involved to make the diagnosis, which is often the case in PACNS [13]. He also had a four vessel cerebral angiogram which revealed anterior cerebral and middle cerebral artery aneurysms, which have been described in the literature in association with PACNS [4].

This represents the first case report of focal PACNS. Glucocorticoid and immunosuppressive therapy are firstline treatments for PACNS based on extrapolations from treatment of other systemic vasculitides and from expert consensus groups, although intravenous immunoglobulin, plasmapharesis, and mycophenolate mofetil have also shown some success [5-7]. Our case describes an unusual presentation of PACNS which had an excellent response to corticosteroid and immunosuppressive therapy.

Acknowledgments Dr. Ho, Ms. Chai, Dr. Hathout, Dr. Mishra, Dr. Yim, Dr. Nishimura report no disclosures. Dr. Vinters is funded by NIH grants \#P50 AG16570, \#P01AG12435, and \#P50 044378, serves on the editorial boards of Human Pathology, Journal of Neuroscience Research, Neuropathology, and Korean Journal of Pathology, and holds stock holdings in 3M, Teva Pharma, Pfizer, and GlaxoSmithKline. Dr. Valdes-Sueiras is funded by NIH grants \# UO1 08-021 and \#UO1 CA 066529. Nikki Yin assisted with preparation of the pathology figures.

\section{Conflict of interest None.}

Open Access This article is distributed under the terms of the Creative Commons Attribution Noncommercial License which permits any noncommercial use, distribution, and reproduction in any medium, provided the original author(s) and source are credited. 
Fig. 2 H\&E stained section of a vessel, cut in longitudinal section, shows polymorphous transmural inflammatory infiltrate between the arrows (a). Note effacement of normal vessel wall by the inflammatory infiltrate (b). Sections of the biopsy immunostained with a marker for $\mathrm{T}$ cells $(\mathrm{CD} 3)$ and $\mathrm{B}$ cells (CD20), indicating that the transmural infiltrate is composed predominantly of $\mathrm{T}$ lymphocytes, with a significant admixture of B lymphocytes $(\mathbf{c}-\mathbf{f})$
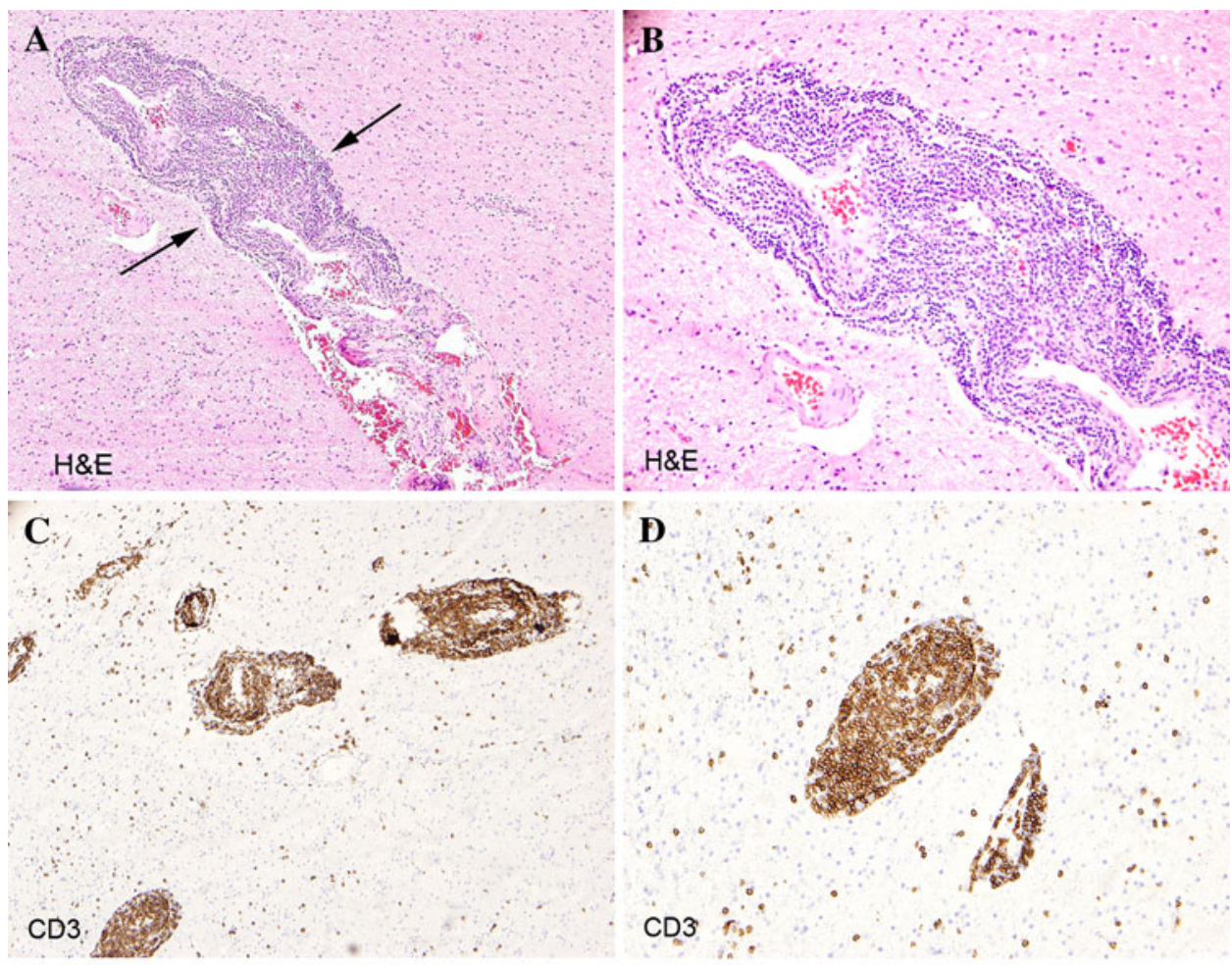

E

F

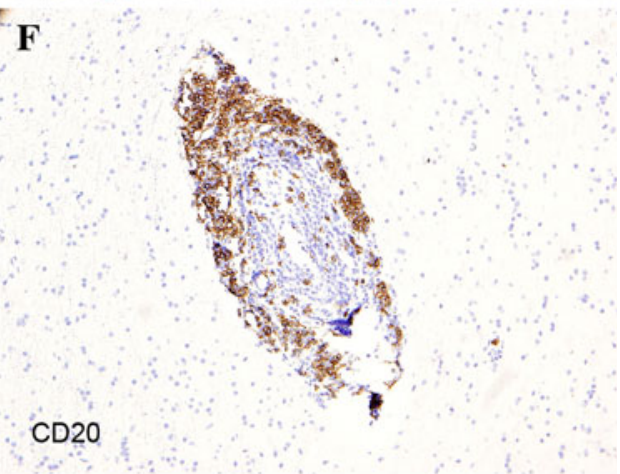

CD20

\section{References}

1. Salvarani C, Brown RD Jr, Calamia KT, Christianson TJ, Weigand SD, Miller DV, Giannini C, Meschia JF, Huston J 3rd, Hunder GG (2007) Primary central nervous system vasculitis: analysis of 101 patients. Ann Neurol 62:442-451

2. Haji-Ali RA, Calabrese LH (2009) Central nervous system vasculitis. Curr Opin Rheumatol 21:10-18

3. Haji-Ali RA (2010) Primary angiitis of the central nervous system: differential diagnosis and treatment. Best Pract Res Clin Rheumatol 24:413-426

4. Nishikawa M, Sakamoto H, Katsuyama J, Hakuba A, Nishimura M (1998) Multiple appearing and vanishing aneurysms: primary angiitis of the central nervous system: case report. J Neurosurg 88:133-137
5. Sen ES, Leone V, Abinun M, Forsyth R, Ramesh V, Friswell M, O'Callaghan F, Ramanan AV (2010) Treatment of primary angiitis of the central nervous system in childhood with mycophenolate mofetil. Rheumatology 49:806-811

6. Euler HH, Schroeder JO, Harten P, Zeuner RA, Gutschmidt HJ (1994) Treatment-free remission in severe systemic lupus erythematosus following synchronization of plasmapheresis with subsequent pulse cyclophosphamide. Arthritis Rheum 37:1784-1794

7. Sherer Y, Levy Y, Langevitz P, Lorber M, Fabrizzi F, Shoenfeld Y (1999) Successful treatment of systemic lupus erythematosus cerebritis with intravenous immunoglobulin. Clin Rheumatol 18:170-173 